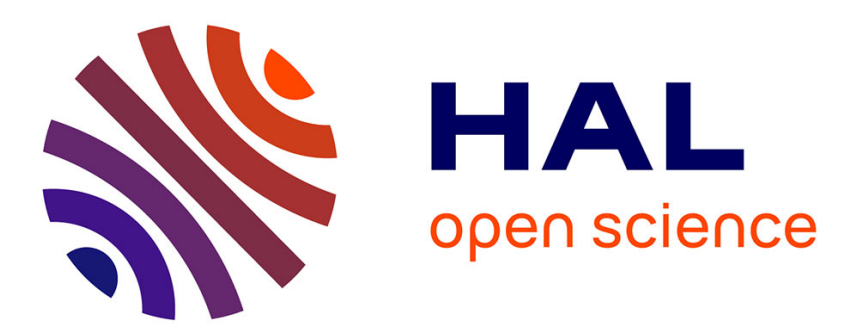

\title{
Kernel Bundle EPDiff: Evolution Equations for Multi-Scale Diffeomorphic Image Registration
}

\author{
Stefan Sommer, François Lauze, Mads Nielsen, Xavier Pennec
}

\section{To cite this version:}

Stefan Sommer, François Lauze, Mads Nielsen, Xavier Pennec. Kernel Bundle EPDiff: Evolution Equations for Multi-Scale Diffeomorphic Image Registration. Scale Space and Variational Methods in Computer Vision, May 2011, Ein-Gedi, Israel. pp.677-688, 10.1007/978-3-642-24785-9_57 . hal00644965

\section{HAL Id: hal-00644965 \\ https://hal.inria.fr/hal-00644965}

Submitted on 25 Nov 2011

HAL is a multi-disciplinary open access archive for the deposit and dissemination of scientific research documents, whether they are published or not. The documents may come from teaching and research institutions in France or abroad, or from public or private research centers.
L'archive ouverte pluridisciplinaire HAL, est destinée au dépôt et à la diffusion de documents scientifiques de niveau recherche, publiés ou non, émanant des établissements d'enseignement et de recherche français ou étrangers, des laboratoires publics ou privés. 


\title{
Kernel Bundle EPDiff: Evolution Equations for Multi-Scale Diffeomorphic Image Registration
}

\author{
Stefan Sommer ${ }^{1}$, François Lauze $^{1}$, Mads Nielsen $^{1,2}$, and Xavier Pennec ${ }^{3}$ \\ 1 Dept. of Computer Science, Univ. of Copenhagen, Denmark \\ sommer@diku.dk \\ 2 Synarc Imaging Technologies, Rødovre, Denmark \\ 3 Asclepios Project-Team, INRIA Sophia-Antipolis, France
}

\begin{abstract}
In the LDDMM framework, optimal warps for image registration are found as end-points of critical paths for an energy functional, and the EPDiff equations describe the evolution along such paths. The Large Deformation Diffeomorphic Kernel Bundle Mapping (LDDKBM) extension of LDDMM allows scale space information to be automatically incorporated in registrations and promises to improve the standard framework in several aspects. We present the mathematical foundations of LDDKBM and derive the KB-EPDiff evolution equations, which provide optimal warps in this new framework. To illustrate the resulting diffeomorphism paths, we give examples showing the decoupled evolution across scales and how the method automatically incorporates deformation at appropriate scales.
\end{abstract}

Keywords: LDDKBM, LDDMM, diffeomorphic registration, scale space, computational anatomy, kernels, momentum

\section{Introduction}

The Large Deformation Diffeomorphic Metric Mapping (LDDMM) framework plays an increasingly important role in image registration for medical image analysis as it provides good registration results along with a solid mathematical foundation allowing meaningful statistics to be computed on the registration results. It has its foundations in the seminal work of Grenander [3] and Christensen et al. [1] together with the theoretical contributions of Dupuis et al. and Trouvé $[2,7]$. The theory in its present state is well described in the paper of Younes et al. [9] and the monograph of Younes [8]. The purpose of this paper is to discuss the mathematical foundation behind a multi scale extension of LDDMM, the Large Deformation Diffeomorphic Kernel Bundle Mapping (LDDKBM), and develop the resulting evolution equations for the registration diffeomorphisms.

The LDDMM construction is based on the concept of kernels which encode the scale of the registration. Coarse to fine approaches, such as used in [4] for nonparametric image registration, can be used as tools to guide the search for the optimal registration but a scale mechanism for LDDMM which is truly consistent with the framework must be linked to the kernels. The role of the kernel and 
deformation at different scales in LDDMM have been addressed by Risser et al. in [5] where the authors propose a multi-kernel approach which constructs new kernel shapes by adding Gaussian kernels. The method effectively changes only the shape of the kernel and does not allow decoupled momentum across scales. To improve the ability of the registration to adapt to scale information, we developed in [6] the LDDKBM extension of LDDMM which allows decoupling of the energy and momentum at each scale, and it therefore enables the algorithm to select the appropriate deformation at each scale individually. An example of an LDDKBM registration is given in Figure 1.

\subsection{Content and Outline}

In the next section, we will summarize the LDDMM framework before providing a detailed account of the mathematical foundation behind the LDDKBM extension. We then progress to developing the KB-EPDiff equations describing the evolution of critical paths in the framework and extending the fundamental EPDiff equations in LDDMM. We will present experiments in Section 5 and conclude in Section 6. The paper thus contributes by

(1) providing a detailed account of the theoretical foundation of the LDDKBM framework for multi scale diffeomorphic registration,

(2) deriving the KB-EPDiff equations which are fundamental for the theoretical understanding of LDDKBM and necessary for practical implementations,

(3) and through examples showing the evolution of diffeomorphism paths governed by the KB-EPDiff equations and how the evolution is decoupled across scales.

\section{The LDDMM Framework}

In the sequel, $\Omega$ will denote a hold-all domain of $\mathbb{R}^{d}(d=2,3$ in applications $)$ and $V$ will denote a Hilbert space of vector fields $v: \Omega \rightarrow \mathbb{R}^{d}$ such that $V$ with associated norm $\|\cdot\|_{V}$ is included in $L^{2}\left(\Omega, \mathbb{R}^{d}\right)$ and admissible as defined in [8, Chap. 9]. Given a time-dependent vector field $t \mapsto v_{t}$ with

$$
\int_{0}^{1}\left\|v_{t}\right\|_{V}^{2} d t<\infty
$$

the associated differential equation $\partial_{t} \varphi_{t}=v_{t} \circ \varphi_{t}$ has with initial condition $\varphi_{s}=\varphi$ a diffeomorphism $\varphi_{s t}^{v}$ as unique solution. The set $G_{V}$ of diffeomorphisms built from $V$ by such differential equations is a Lie group, and $V$ is its tangent space at each point. The inner product on $V$ associated to the norm $\|\cdot\|_{V}$ makes $G_{V}$ a Riemannian manifold with right-invariant metric. Setting $\varphi_{00}^{v}=I d_{\Omega}$, the map $t \mapsto \varphi_{0 t}^{v}$ is a path from $\operatorname{Id}_{\Omega}$ to $\varphi$ with energy given by (1). A critical path for the energy is a geodesic on $G_{V}$.

In the LDDMM framework, registration is performed through the action of diffeomorphisms in $G_{V}$ on geometric objects. This approach is very general 


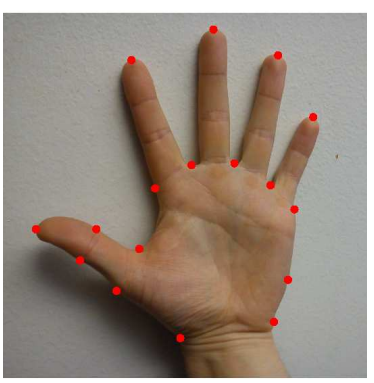

(a) Moving hand and landmarks (red)

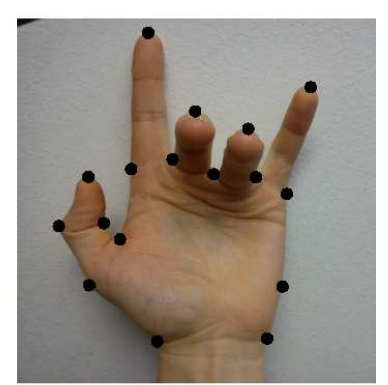

(b) Fixed hand and landmarks (black)

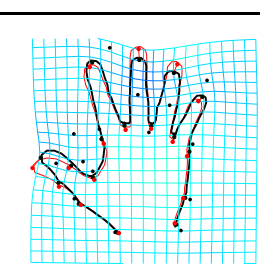

(c) $\mathrm{t}=0.25$

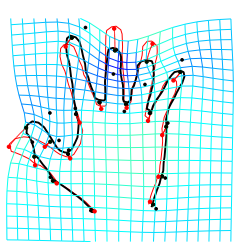

(d) $\mathrm{t}=0.50$

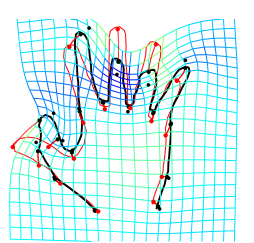

(e) $\mathrm{t}=0.75$

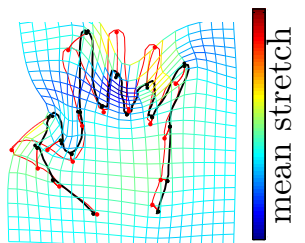

(f) $t=1.00$

Fig. 1. Matching landmarks of hand (a) to landmarks of hand (b) with LLDKBM multiscale registration with Gaussian kernels of four scales. The critical path of diffeomorphisms determined by the KB-EPDiff equations derived in this paper is shown for four time steps (c)-(f) along with the outline of hand (a) (red line) and deformation of an initially square grid. Black curve shows the transported outline, and the grids are colored with the trace of Cauchy-Green strain tensor (log-scale). As we will see in Section 5, multiple scales are necessary to properly match the hands and movement occur decoupled across scales for the critical path shown.

and allows the framework to be applied to both landmarks, curves, surfaces, images, and tensors. In the case of landmarks, the action of a diffeomorphism $\varphi$ takes the form $\varphi \cdot x=\varphi(x)$, and given landmarks $x_{1}, \ldots, x_{N}$ and $y_{1}, \ldots, y_{N}$, the registration amounts to a search for $\varphi$ such that $\varphi \cdot x_{i} \sim y_{i}$ for all $i=1, \ldots, N$. In exact matching, we wish $\varphi \cdot x_{i}$ be exactly equal to $y_{i}$ but, more frequently, we allow some amount of inexactness to account for noise and give smoother diffeomorphisms. This is done by defining a quality of match measure $U$ and a regularization measure $E_{1}$ to give a combined energy

$$
E(\varphi)=E_{1}(\varphi)+\lambda U(\varphi)
$$

Here $\lambda$ is a positive real representing the trade-off between regularity and goodness of fit and $U$ is often the $L^{2}$-error which in the landmark case takes the form $U(\varphi)=\sum_{i=1}^{N}\left\|\varphi\left(x_{i}\right)-y_{i}\right\|^{2}$. The regularization term $E_{1}$ is defined as

$$
E_{1}(\varphi)=\min _{v_{t} \in V, \varphi_{01}^{v}=\varphi} \int_{0}^{1}\left\|v_{s}\right\|_{V}^{2} d s .
$$


It penalizes highly varying paths and, therefore, a low value of $E_{1}(\varphi)$ implies that $\varphi$ is regular.

The regularity is ultimately controlled by the norm on $V$ and this norm is associated to a reproducing kernel $K: \Omega \times \Omega \rightarrow \mathbb{R}^{d \times d}$. The kernel is often chosen to ensure rotational and translational invariance [8] and the Gaussian kernel $K(x, y)=\exp \left(\frac{\|x-y\|^{2}}{\sigma^{2}}\right) \operatorname{Id}_{d}$ is a convenient and often used choice. The scaling factor $\sigma$ is not limited to Gaussian kernels and allows for many kernels to vary the amount of regularization. Larger scales lead in general to higher regularization and smoother diffeomorphisms, whereas smaller kernels penalize higher frequencies less and often gives better matches. This phenomenon is in particular apparent for objects with sparse information and images with e.g. areas of constant intensity.

\section{Kernel, Momentum and LDDKBM}

The Large Deformation Diffeomorphic Kernel Bundle Mapping (LDDKBM) framework extends LDDMM by equipping diffeomorphism manifolds $G_{V}$ in LDDMM with vector bundles allowing deformation to be described at different scales. We start this section by discussing the relation between kernels and momentum in LDDMM before giving details on the mathematical foundation of LDDKBM.

\subsection{Kernel and Momentum}

The admissibility of $V$ implies that for any $x \in \Omega$, the evaluation $\delta_{x}: v \mapsto$ $v(x) \in \mathbb{R}^{d}$ is well-defined and continuous. Thus, for any $a \in \mathbb{R}^{d}$ the map $a \otimes \delta_{x}$ : $v \mapsto a^{T} v(x)$ belongs to the topological dual $V^{*}$ of $V$ implying the existence of the kernel $K: \Omega \times \Omega \rightarrow \mathbb{R}^{d \times d}$ so that, for any constant vector $a \in \mathbb{R}^{d}$, the vector field $K(\cdot, x) a \in V$ represents $a \otimes \delta_{x}$ and $\langle K(\cdot, x) a, K(\cdot, y) b\rangle_{V}=a^{T} K(x, y) b$ for all points $x, y \in \Omega$ and all vectors $a, b \in \mathbb{R}^{d}$. This latter property is denoted the reproducing property and gives $V$ the structure of a reproducing kernel Hilbert space (RKHS). Tightly connected to the norm and kernels is the notion of momentum given by the linear momentum operator $L: V \rightarrow L^{2}\left(\Omega, \mathbb{R}^{d}\right)$ which satisfies

$$
\langle L v, w\rangle_{L^{2}\left(\Omega, \mathbb{R}^{d}\right)}=\int_{\Omega}(L v(x))^{T} w(x) d x=:\langle v, w\rangle_{V}
$$

for all $v, w \in V$. The momentum operator connects the inner product on $V$ with the inner product in $L^{2}\left(\Omega, \mathbb{R}^{d}\right)$, and the image $L v$ of an element $v \in V$ is denoted the momentum of $v$. The reader can consult [8] for a thorough introduction to reproducing kernels, especially with a view towards the LDDMM framework.

\subsection{The Kernel Bundle and LDDKBM}

The LDDMM framework is limited to the choice of only one kernel shape and scale but deformation on different scales are often needed for good registration. 
To address this problem, we constructed in [6] a multi scale extension of LDDMM resulting in the LDDKBM framework.

In order to use more kernels, we consider a parameter set $I_{W}$ and subspaces $V_{r}, r \in I_{W}$ of the tangent space $V$ where each $V_{r}$ is equipped with a norm $\|\cdot\|_{r}$, corresponding kernel $K_{r}$, and momentum operator $L_{r}$. Typically, $I_{W}$ will be a discrete set or a closed and bounded interval of $\mathbb{R}^{+}$representing different scales. We then let $W$ be the space of functions $w: I_{W} \rightarrow V, w_{r} \in V_{r}$ such that

$$
\int_{I_{W}}\left\|w_{r}\right\|_{r}^{2} d r<\infty \text { and } \int_{I_{W}}\left\|w_{r}\right\|_{r} d r<\infty .
$$

The vector space structures on $V_{r}$ induce a vector space structure on $W$, and it can be shown that under reasonable assumptions, the inner product

$$
\langle v, w\rangle_{W}=\int_{I_{W}}\left\langle v_{r}, w_{r}\right\rangle_{r} d r, v, w \in W
$$

turns $W$ into a Hilbert space. Moreover the integral $\Psi(w)=\int_{I} w_{r} d r$ is well defined for $w \in W$ and allows us to pass from $W$ to $V$. With this construction, we obtain a vector bundle $G_{V} \times W$, the kernel bundle, allowing kernels of different sizes and shapes, and a map $G_{V} \times W \rightarrow T G_{V}=G_{V} \times V$ that provides an extension of $T G_{V}$ to multiple scales.

Using $\Psi$ we can connect time varying paths $w_{t}=\left\{w_{t, r}\right\}_{r}$ in $W$ and paths on the manifold $G_{V}$ by

$$
w_{t} \mapsto \varphi_{0 t}^{\Psi(w)} .
$$

The path energy is in LDDKBM measured using the norm on $W$, i.e. we define the energy

$$
E_{1}^{W}\left(w_{t}\right)=\int_{0}^{1}\left\|w_{s}\right\|_{W}^{2} d s .
$$

which induces a regularization measure on diffeomorphisms

$$
E_{1}^{W}(\varphi)=\min _{w_{t} \in W, \varphi_{01}^{\Psi(w)}=\varphi} \int_{0}^{1}\left\|w_{s}\right\|_{W}^{2} d s
$$

Together with a quality of match measure $U(\varphi)$, this allows a reformulation of the registration problem as the search for a diffeomorphism minimizing

$$
E^{W}(\varphi)=E_{1}^{W}(\varphi)+\lambda U(\varphi) .
$$

The above formulation should be compared with the standard LDDMM formulation using the regularization (2), and it is immediately clear that the standard LDDMM method is the special case with only one scale and hence $W=V$.

It is interesting to note that $W$ possesses a structure very similar to a RKHS. On $V$ we have for each $x \in \Omega$ and $a \in \mathbb{R}^{d}$ the evaluation functional $a \otimes \delta_{x}(v)=$ $a^{T} v(x)$. Using the integral map $\Psi$ defined above, we define the linear maps on $W$

$$
a \otimes \delta_{x}^{\Psi}(w):=\int_{I_{W}} a \otimes \delta_{x}\left(w_{r}\right) d r=\int_{I_{W}} a^{T} w_{r}(x) d r=a \otimes \delta_{x}(\Psi(w))
$$


As seen from the equation, the maps evaluate $w_{r}$ at each scale and integrate the results using $\Psi$. These maps are continuous and hence in the dual $W^{*}$. For the elements $K(\cdot, x) a=\left\{K(\cdot, x)_{r} a\right\}_{r} \in W$, we have

$$
\begin{gathered}
\langle K(\cdot, x) a, K(\cdot, y) b\rangle_{W}=\int_{I_{W}}\left\langle K(\cdot, x)_{r} a, K(\cdot, y)_{r} b\right\rangle_{r} d r=\int_{I_{W}} a^{T} K_{r}(x, y) b d r \\
=a^{T} \int_{I_{W}} K_{r}(x, y) b d r=a \otimes \delta_{x}^{\Psi}(K(\cdot, y) b)=a^{T} \Psi(K(x, y) b)
\end{gathered}
$$

which is similar to the reproducing property for LDDMM except for the integration performed by $\Psi$ on the right-hand side of the equation. Also, close to the RKHS situation, we see that

$\langle K(\cdot, x) a, w\rangle_{W}=\int_{I_{W}}\left\langle K(\cdot, x)_{r} a, w_{r}\right\rangle_{r} d r=\int_{I_{W}} a^{T} w_{r}(x) d r=a \otimes \delta_{x}^{\Psi}(w), w \in V$ again with the integration of $w$ occuring in $a \otimes \delta_{x}^{\Psi}(w)$.

\section{EPDiff and KB-EPDiff}

The EPDiff equations in LDDMM describes the evolution of optimal paths for the registration problem. They are most often formulated in the following form: let $a_{t}=L v_{t}$ denote the momentum at time $t$ and assume that $\varphi_{t}$ is a path minimizing $E_{1}(\varphi)$ with $\varphi_{1}=\varphi$ minimizing $E(\varphi)$ and $v_{t}$ is the derivative of $\varphi_{t}$. Then $v_{t}$ satisfies the system

$$
v_{t}=\int_{\Omega} K(\cdot, x) a_{t}(x) d x, \quad \frac{d}{d t} a_{t}=-D a_{t} v_{t}-a_{t} \nabla \cdot v_{t}-\left(D v_{t}\right)^{T} a_{t} .
$$

The first equation connects the momentum $a_{t}$ with the velocity $v_{t}$, and the second describes the evolution of the momentum. The EPDiff equations can be interpreted as geodesic equations on the manifold $G_{V}$ and are important for implementations since we can limit the search for optimal paths to paths satisfying the system.

As we will show in this section, there exists similar equations for LDDKBM: if $\Psi\left(w_{t}\right)$ is the derivative of the path of diffeomorphisms $\varphi_{t}$ minimizing (4) with $\varphi=\varphi_{1}$ minimizing (5) then

$$
\begin{aligned}
& w_{r, t}=\int_{\Omega} K_{r}(\cdot, x) a_{r, t}(x) d x, \\
& \frac{d}{d t} a_{r, t}=\int_{I_{W}}-D a_{r, t} w_{s, t}-a_{r, t} \nabla \cdot w_{s, t}-\left(D w_{s, t}\right)^{T} a_{r, t} d s .
\end{aligned}
$$

with $a_{r, t}$ being the momentum for the part $w_{r, t}$ of $w_{t}$. In essence, the standard EPDiff equations are integrated over the parameter space $I_{W}$ to obtain the evolution of the momentum at each scale, and, in particular, the result will imply that the momentum conservation property of LDDMM also holds in LDDKBM. We will derive the KB-EPDiff equations in a more general form which implies the above formulation, and, for doing this, we will follow the strategy in [8] for the LDDMM case. 


\subsection{Euler-Lagrange equations}

For any time varying path $w_{t}$ in $W$, we denote by $\varphi_{t_{1} t_{2}}^{\Psi(w)}$ the diffeomorphism obtained by integrating $\Psi\left(w_{t}\right)$ from time $t_{1}$ to time $t_{2}$. The end of the integrated path $\varphi_{01}^{\Psi(w)}$ is the diffeomorphism used for the registration. For the energy $E^{W}\left(w_{t}\right)=E^{W}\left(\varphi_{01}^{\Psi(w)}\right)$, we consider a variation $h_{t} \in W$ and calculate

$$
\frac{d}{d \epsilon} E\left(w_{t}+\epsilon h_{t}\right)=2 \int_{0}^{1}\left\langle w_{t}, h_{t}\right\rangle_{W} d t+\frac{d}{d \epsilon} U\left(\varphi_{01}^{\Psi(w)+\epsilon \Psi(h)}\right) .
$$

Following [8], we define $\operatorname{Ad}_{\varphi} v(x)=(D \varphi v) \circ \varphi^{-1}(x)$ for $v \in V$ and get a functional $\operatorname{Ad}_{\varphi}^{*}$ on the dual $V^{*}$ of $V$ by $\left(\operatorname{Ad}_{\varphi}^{*} \rho \mid v\right)=\left(\rho \mid \operatorname{Ad}_{\varphi}(v)\right)$. It is shown in [8] that a variation $\tilde{h}_{t}$ in $V$ of the match functional satisfies

$$
\frac{d}{d \epsilon} U\left(\varphi_{01}^{v+\epsilon \tilde{h}}\right)=\int_{0}^{1}\left(\operatorname{Ad}_{\varphi_{t 1}^{v}}^{*} \bar{\partial} U\left(\varphi_{01}^{v}\right) \mid \tilde{h}_{t}\right) d t
$$

with $\bar{\partial} U$ denoting the Eulerian differential of $U$ (see [8, Chap. 10]). Inserting into (7) gives

$$
\frac{d}{d \epsilon} E\left(w_{t}+\epsilon h_{t}\right)=2 \int_{0}^{1}\left\langle w_{t}, h_{t}\right\rangle_{W} d t+\int_{0}^{1}\left(\operatorname{Ad}_{\varphi_{t 1}^{\Psi(w)}}^{*} \bar{\partial} U\left(\varphi_{01}^{\Psi(w)}\right) \mid \Psi\left(h_{t}\right)\right) d t .
$$

For each $r$, we define the operator $\operatorname{Ad}_{\varphi}^{T, r} v=K_{r}\left(\operatorname{Ad}_{\varphi}^{*}\left(L_{r} v\right)\right)$ which then satisfies $\left\langle\operatorname{Ad}_{\varphi}^{T, r} v, w\right\rangle_{r}=\left(\operatorname{Ad}_{\varphi}^{*}\left(L_{r} v\right) \mid w\right)$, and we can now derive the fundamental results [8, Prop. 11.6/Cor. 11.7] in the LDDKBM case:

Proposition 1. If $w_{t}$ is an optimal path for $E^{W}$ then for almost every $r \in I_{W}$,

$$
w_{t, r}=\operatorname{Ad}_{\varphi_{t 1}^{\Psi}}^{T, r} w_{1, r}
$$

with $w_{1, r}=-\frac{1}{2} \nabla^{V_{r}} U\left(\varphi_{01}^{\Psi(w)}\right)$.

Proof. Assume instead that there exists a time varying $h_{t}$ in $W$ and $t \in[0,1]$ such that

$$
\begin{aligned}
0 & <\int_{I_{W}}\left\langle w_{t, r}-\operatorname{Ad}_{\varphi_{t 1}^{\Psi(w)}}^{T, r} w_{1, r}, h_{t, r}\right\rangle_{r} d r=\int_{I_{W}}\left\langle w_{t, r}, h_{t, r}\right\rangle_{r} d r-\int_{I_{W}}\left\langle\operatorname{Ad}_{\varphi_{t 1}^{\Psi(w)}}^{T, r} w_{1, r}, h_{t, r}\right\rangle_{r} d r \\
& =\left\langle w_{t}, h_{t}\right\rangle+\frac{1}{2} \int_{I_{W}}\left(\operatorname{Ad}_{\varphi_{t 1}^{\Psi(w)}}^{*} \bar{\partial} U\left(\varphi_{01}^{\Psi(w)}\right) \mid h_{t, r}\right) d r \\
& =\left\langle w_{t}, h_{t}\right\rangle+\frac{1}{2}\left(\operatorname{Ad}_{\varphi_{t 1}^{\Psi(w)}}^{*} \bar{\partial} U\left(\varphi_{01}^{\Psi(w)}\right) \mid \Psi\left(h_{t}\right)\right) .
\end{aligned}
$$

But the right hand side vanishes for all $t$ and all $h_{t}$ by (8) and the fact that $w_{t}$ is optimal for $E^{W}$, a contradiction.

Corollary 1. Under the same conditions, for almost every $r \in I_{W}$,

$$
w_{t, r}=\operatorname{Ad}_{\varphi_{t 0}^{\Psi}}^{T, r} w_{0, r} .
$$

The proof of the corollary is identical to the proof of [8, Cor. 11.7]. 


\subsection{Scale Conservation and KB-EPDiff}

In LDDKBM, the momentum of a path in general differ across scales. For a path $w_{t}$ in $W$, we let $a_{t}$ be the bundle momentum defined by $a_{t, r}=L_{r}\left(w_{t, r}\right)$ recalling that $L_{r}$ is the momentum operator at scale $r$. For each $t$, we can consider $a_{t}$ to be in the dual $W^{*}$ by $\left(a_{t} \mid \tilde{w}\right)=\int_{I_{W}}\left(a_{t, r} \mid \tilde{w}_{r}\right) d r$ which is continuous since

$$
\left|\left(a_{t} \mid \tilde{w}\right)\right| \leq\left|\int_{I_{W}}\left(a_{t, r} \mid \tilde{w}_{r}\right) d r\right|=\left|\int_{I_{W}}\left\langle w_{t, r}, \tilde{w}_{r}\right\rangle_{r} d r\right| \leq\left\|w_{t}\right\|\|\tilde{w}\| .
$$

Suppose now $w_{t}$ satisfies the transport equation (9) for almost every $r \in I_{W}$. Then for all $\tilde{w} \in W$,

$$
\begin{aligned}
\left(a_{t} \mid \tilde{w}\right) & =\int_{I_{W}}\left\langle w_{t, r}, \tilde{w}_{r}\right\rangle_{r} d r=\int_{I_{W}}\left\langle\operatorname{Ad}_{\varphi_{t 0}^{\Psi(w)}}^{T, r} w_{0}, \tilde{w}_{r}\right\rangle_{r} d r \\
& =\int_{I_{W}}\left\langle w_{0, r}, \operatorname{Ad}_{\varphi_{t 0}^{\Psi(w)}} \tilde{w}_{r}\right\rangle_{r} d r=\left(a_{0} \mid \operatorname{Ad}_{\varphi_{t 0}^{\Psi(w)}} \tilde{w}\right)
\end{aligned}
$$

where $\operatorname{Ad}_{\varphi_{t 0}^{\Psi(w)}} \tilde{w}$ is the element of $W$ obtained by applying $\operatorname{Ad}_{\varphi_{t 0}^{\Psi(w)}}$ to each $\tilde{w}_{r}$. The above equation shows that the momentum at time $t$ is completely specified by the momentum at time 0 and thus reproduces the momentum conservation property for LDDMM. Note that since $\tilde{w}$ can be chosen arbitraly in (10), the momentum is conserved for each scale separately. By differentiating $\operatorname{Ad}_{\varphi_{t 0}^{\Psi(w)}} \tilde{w}$, the momentum conservation property directly implies the equation

$$
\partial_{t}\left(a_{t} \mid \tilde{w}\right)=-\left(a_{t} \mid D \Psi\left(w_{t}\right) \tilde{w}-D \tilde{w} \Psi\left(w_{t}\right)\right)
$$

or, equivalently,

$$
\partial_{t} a_{t}+\operatorname{ad}_{\Psi\left(w_{t}\right)}^{*} a_{t}=0
$$

with $\left(\operatorname{ad}_{\Psi\left(w_{t}\right)}^{*} a_{t} \mid \tilde{w}\right)=\left(a_{t} \mid D \Psi\left(w_{t}\right) \tilde{w}-D \tilde{w} \Psi\left(w_{t}\right)\right)$. Both equations imply the system (6) and extend the EPDiff equations for LDDMM. We denote them KBEPDiff.

\subsection{KB-EPDiff for Landmarks: An Example}

To give a concrete application of the KB-EPDiff equations, we redo the calculation for LDDMM landmark matching with scalars kernels to arrive at the corresponding system for LDDKBM. The initial momentum $a_{0, r}$ will in this case be supported at the $N$ landmarks $x_{i}, i=1 \ldots, N$, i.e. $a_{0, r}=\sum_{i=1}^{N} a_{0, r, i} \otimes \delta_{x_{i}}$ with vectors $a_{0, r, i} \in \mathbb{R}^{d}$. We let $x_{t, i}$ denote the trajectory of the $i$ th landmark so that $x_{t, i}=\varphi_{0 t}^{\Psi(w)}\left(x_{0, i}\right)$.

Letting $a_{t, r, i}=\left(D \varphi_{t 0}^{\Psi(w)}\right)^{T} a_{0, r, i}$, we get from (10)

$$
\begin{aligned}
\left(a_{t, r} \mid \tilde{w}\right) & =\left(\operatorname{Ad}_{\varphi_{t 0}^{\Psi(w)}}^{*}\left(\sum_{i=1}^{N} a_{0, r, i} \otimes \delta_{x_{0, i}}\right) \mid \tilde{w}\right)=\left(\sum_{i=1}^{N} a_{0, r, i} \otimes \delta_{x_{0, i}} \mid \operatorname{Ad}_{\varphi_{t 0}^{\Psi(w)}}(\tilde{w})\right) \\
& =\sum_{i=1}^{N} a_{0, r, i}^{T}\left(D \varphi_{t 0}^{\Psi(w)} \tilde{w}\right) \circ \varphi_{0 t}^{\Psi(w)}\left(x_{0, i}\right)=\left(\sum_{i=1}^{N} a_{t, r, i} \otimes \delta_{x_{t, i}} \mid \tilde{w}\right) .
\end{aligned}
$$


Since $\frac{d}{d t}\left(D_{x_{t, i}} \varphi_{t 0}^{\Psi(w)}\right)^{T}=-D_{x_{t, i}} \Psi\left(w_{t}\right)^{T}\left(D_{x_{0, i}} \varphi_{t 0}^{\Psi(w)}\right)^{T}$, the derivative of the momentum satisfies

$$
\frac{d}{d t} a_{t, r, i}=\frac{d}{d t}\left(\left(D \varphi_{t 0}^{\Psi(w)}\right)^{T} a_{0, r, i}\right)=-D_{x_{t, i}} \Psi\left(w_{t}\right)^{T} a_{t, r, i}
$$

We therefore have the trajectory of the landmarks and momentum evolution completely described by the system

$$
\begin{aligned}
& \Psi\left(w_{t}\right)=\int_{I_{W}} \sum_{l=1}^{N} K_{r}\left(\cdot, x_{t, l}\right) a_{t, r, l} d r \\
& \frac{d}{d t} a_{t, r, i}=-\left(\int_{I_{W}} \sum_{l=1}^{N} D_{1}\left(K_{s}\left(x_{t, i}, x_{t, l}\right) a_{t, s, l}\right)^{T} d s\right) a_{t, r, i} \\
& x_{t, i}=\varphi_{0 t}^{\Psi(w)}\left(x_{0, i}\right) .
\end{aligned}
$$

Note that the system is finite if $I_{W}$ is finite.

\section{Experiments}

We perform two experiments showing the progressing deformation as we move along the critical path of the LDDKBM energy functional specified by the KBEPDiff equations and showing the different deformation across scale. The first experiment is performed on landmarks from images of hands and the second on a simpler and artificial example to better visualize the scale differences.

\subsection{Hand Outlines}

We first consider the hand outlines shown in Figure 1 and Figure 2. Using the landmarks (red dots) on the moving hand image, we wish to compute the LDDKBM match against the landmarks on the fixed image (black dots). The match is computed with three scales of 8,4 , and 2 units of the grid overlayed the figures. After optimizing for the optimal registration, we show in Figure 1 the progression of the deformation as we move along the critical path. The final deformation occurs rightmost for $t=1$. The initially square grid is seen to progressively deform as time increases and the outline is moved to match the outline of the fixed image.

Figure 2 shows the results of computing the same match with standard LDDMM with each of the three scales as well as the final match from LDDKBM repeated for comparison. For LDDMM with the largest scale, the match is poor and the sharp bend of the thumb is especially badly modelled. The situation improves for the middle scale though the bend of the thumb is still not sufficiently sharp and the match is bad for the middle fingers. For the smallest scales, the thumb is correctly matched but now the smaller scale is not able to model the even movement of the index finger. The LDDKBM method is by including all scales able to correctly register all the critical areas, and, at the same time, it gives the best match of the landmarks. 

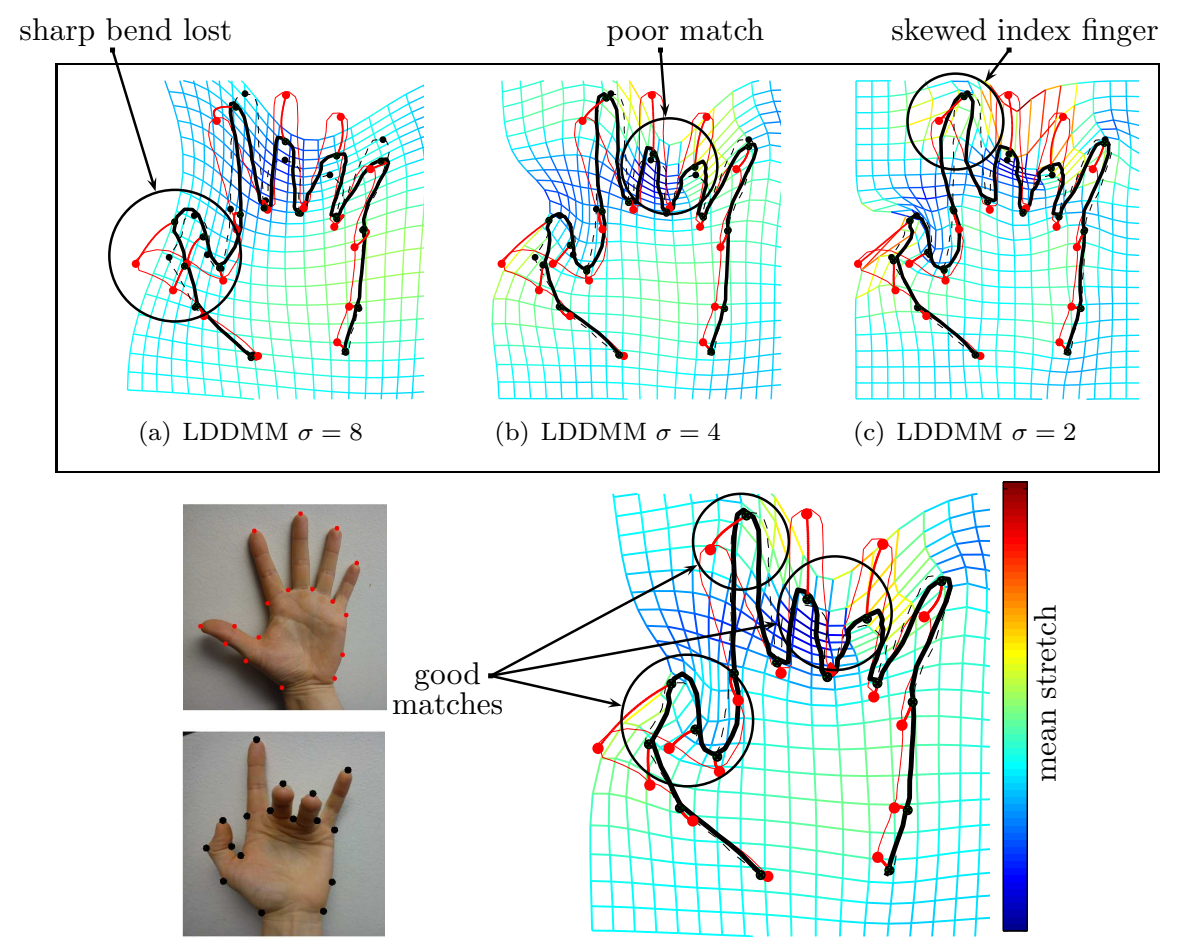

$\begin{array}{ll}\text { (d) Moving hand (top), fixed } & \text { (e) LDDKBM }\end{array}$

Fig. 2. Matching the hands of Figure 1 and shown in (d) for three scales of LDDMM and LDDKBM. The red landmarks of the moving hand are matched against the black landmarks of the fixed hand with the green crossed points showing the result of the match. The outline of the moving hand (red line) is transported to the black outline and should be compared with the outline of the fixed image (black dashed). The LDDKBM method is able to correctly match all the critical areas on which LDDMM fails, see text.

\subsection{KB-EPDiff Across Scales}

To show how LDDKBM decouples deformation across scale, we extend the experiment presented in [6] where four points (red) are matched to four points (black) with results (green crossed) using LDDKBM with three scales. In Figure 3, the result of the registration is visible in the top right subfigure and the evolution of the critical path generated by the KB-EPDiff equations is shown with time increasing across columns. For the lower rows, the deformation at each scale is here shown independently. We see how most of the transport occurs at the largest scale with the middle scale participating to some degree and starting the acceleration of the two points having to move the farthest. The lowest scale perform almost no horizontal movement but takes care of the fine adjustment allowing 


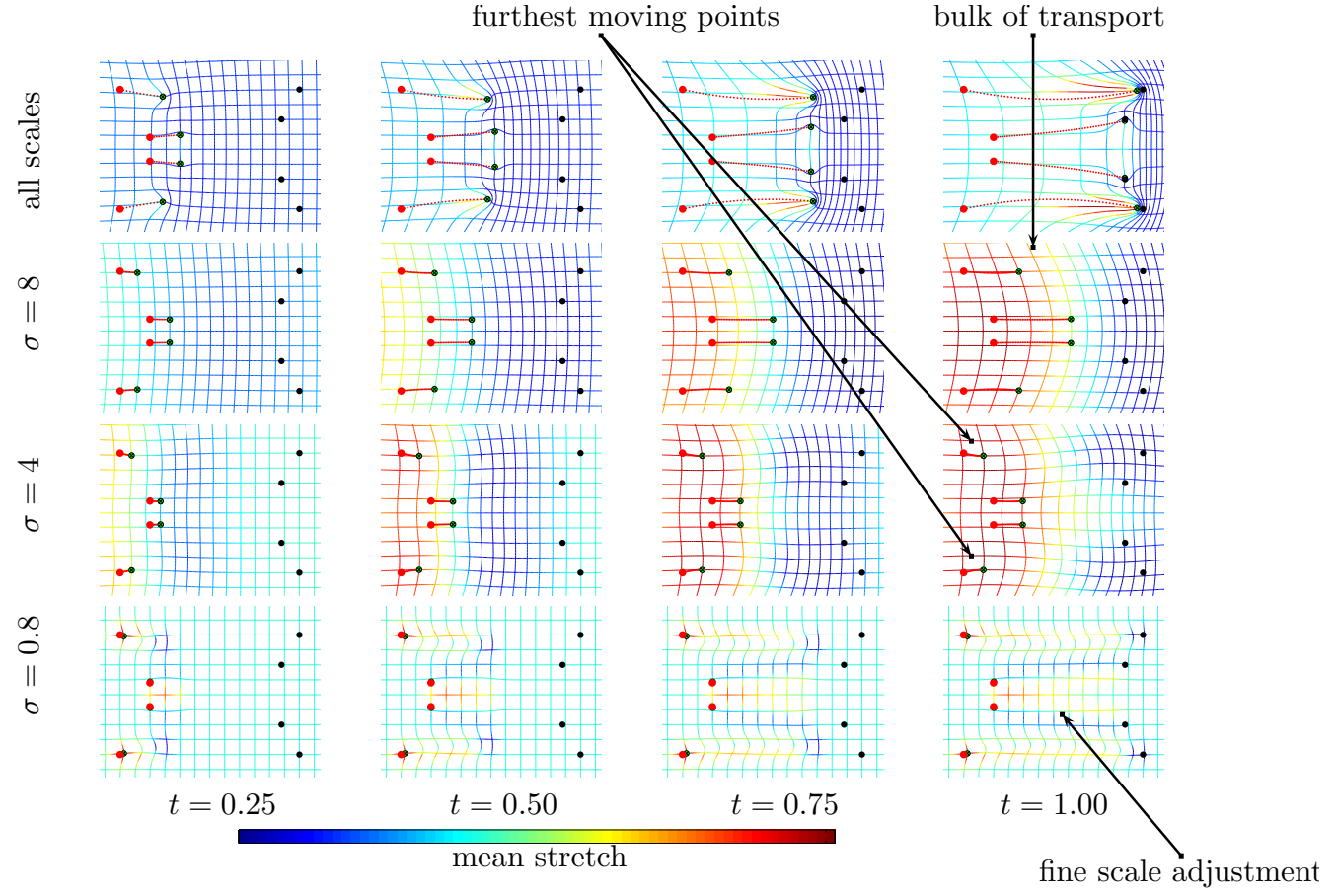

Fig. 3. LDDKBM match of four landmarks (red) to four landmarks (black) with results (green, crossed) for Gaussian kernels of three scales. Top row: critical path determined by KB-EPDiff equations, row 2-4: individual contribution of each of the three scales (scale $\sigma$ in grid units). The columns shows four time points of the critical path with the rightmost being the final deformation. Initially square grids are shown deformed by the diffeomorphism, and the grids are colored with the trace of Cauchy-Green strain tensor indicative of the mean stretch (log-scale for each row individually). The largest scales contribute to most of the transport movement with smooth deformations while the smallest scale performs fine adjustment of the trajectories to obtain a good match.

the LDDKBM method to achieve an arguably superior registration compared to the corresponding LDDMM registrations which can be found in [6].

\section{Conclusion}

We have detailed the mathematical foundation behind the LDDKBM framework for registration which extends LDDMM to include deformation at multiple scales. This includes deriving the KB-EPDiff equations describing the evolution of critical paths in the framework, and the resulting differential systems give insight into the geometry behind the framework in addition to being essential for algorithms for computing the improved registrations. We have provided examples showing the evolution governed by the KB-EPDiff equations and how 
the deformation differ across scales as well as showing the superior registration quality of the LDDKBM method on real images.

A further understanding of the structures behind LDDKBM may allow improved discretization and computational schemes to be developed. Therefore, we expect to look more into the geometry behind the vector bundle construction of LDDKBM and relate the energy to geometric notions generalizing e.g. Riemannian metrics to vector bundles on manifolds.

\section{References}

1. Christensen, G., Rabbitt, R., Miller, M.: Deformable templates using large deformation kinematics. Image Processing, IEEE Transactions on 5(10), 1435-1447 (2002)

2. Dupuis, P., Grenander, U., Miller, M.I.: Variational problems on flows of diffeomorphisms for image matching (1998)

3. Grenander, U.: General Pattern Theory: A Mathematical Study of Regular Structures. Oxford University Press, USA (Feb 1994)

4. Modersitzki, J., Haber, E.: Cofir: Coarse and fine image registration. Real-Time PDE-Constrained Optimization p. 30322 (2004)

5. Risser, L., Vialard, F., Wolz, R., Holm, D.D., Rueckert, D.: Simultaneous fine and coarse diffeomorphic registration: application to atrophy measurement in alzheimer's disease. MICCAI 2010 13(Pt 2), 610-617 (2010), PMID: 20879366

6. Sommer, S., Nielsen, M., Lauze, F., Pennec, X.: A Multi-Scale kernel bundle for LDDMM: towards sparse deformation description across space and scales. In: IPMI 2011. Springer (2011)

7. Trouvé, A.: An infinite dimensional group approach for physics based models in patterns recognition (1995)

8. Younes, L.: Shapes and Diffeomorphisms. Springer (2010)

9. Younes, L., Arrate, F., Miller, M.I.: Evolutions equations in computational anatomy. NeuroImage 45(1, Supplement 1), S40-S50 (Mar 2009) 